\title{
Wolf-Rayet Stars in the Old Days
}

\author{
Leonard V. Kuhi \\ University of Minnesota \\ 213 Morrill Hall \\ 100 Church St. S.E. \\ Minneapolis, Minnesota 55455
}

This morning, as part of the welcoming ceremonies opening this conference, we were reminded that Indonesia consists of over 13,000 islands and that the coat of arms carries the phrase: "Unity in Diversity." We might take the same theme for the study of Wolf-Rayet Stars. There has certainly been no shortage of diversity over the 120 years since their discovery and we all keep hoping for unity; perhaps IAU Symposium No. 143 will provide it! The conference was opened this morning with three strokes of the ceremonial gong to chase away all the bad spirits. So far that symbolic action has been most successful but perhaps more likely due to the kind hospitality of our hosts and the special warmth of Bali. We owe them all a vote of thanks!

What does the title of this talk: "Wolf-Rayet Stars in the Old Days" actually mean? My first reaction was that it reflects a sign of old age when one is invited to be the after dinner speaker. After all, only "old" astronomers are afforded that pleasure if memory serves me. Perhaps the title means that the study of Wolf-Rayet stars is a mature subject and that there are actually old days to talk about. Or perhaps it goes back to my old work on Wolf-Rayet stars in the 1960's but that would be vanity. More likely it goes back to two conferences held in 1968 and 1971. The first was held at Boulder and was simply entitled "Wolf-Rayet Stars"; the second was the IAU Symposium No. 49 held in Buenos Aires on "Wolf-Rayet and High Temperature Stars." The Boulder symposium rekindled interest in Wolf-Rayet stars with several major presentations: Lindsey Smith (1969) on classification, luminosities, binary nature and galactic distribution; L. V. Kuhi (1969) on continuous energy distributions, spectral scans, line profiles and infrared observations; A. B. Underhill (1969) on spectroscopic diagnostics and atmospheric models; and R. N. Thomas (1969) on the physical structure of Wolf-Rayet stars.

The Buenos Aires conference in 1971 reflected a two year period of intense activity following up on many of the problems discussed at Boulder. R. N. Thomas (1971) started off the symposium with an overview of the general problems of extended atmospheres and non- 
classical stellar atmospheric models. Major theoretical presentations were made by D. Van Blerkom (1971) on the theory of Wolf-Rayet spectra and by B. Paczynski (1971) on evolutionary aspects of Wolf-Rayet stars. L. F. Smith (1971) reviewed the classification and galactic distribution of Wolf-Rayet stars, the interpretation of the WN sequence and of the WC stars. L. V. Kuhi (1971) discussed Wolf-Rayet binaries and used the observed changes in C III and IV line profiles to determine the atmospheric stratification and run of temperature with radius. Much new information was provided at that meeting which basically set the stage for the ensuing research of the last twenty years. It is gratifying today to see how much of that original work has withstood the test of time and the onslaught of countless young Ph.D.'s all eager to publish and not perish.

But we really shouldn't get too smug in our reflections about the recent past; after all twenty years can hardly be "the old days." Also, as Peter Conti remarked earlier: "The old astronomers twenty years ago looked so much older than the old astronomers do today." Therefore, I will take as the crucial timescale to "the old days" as 50 years -- my lifetime to astrophysical accuracy. It is only then that we come to the real pioneers of the field, namely those astronomers who published before 1940.

Van der Hucht in his opening remarks has already referred to the discovery of WolfRayet stars by Wolf and Rayet (1867) who had conducted a visual survey of the spectra of stars in Cygnus. They noted that three stars, HD 191765, 192103 and 192641, showed bright emission bands and colors which were "all yellow, orange yellow and greenish yellow." More such stars were discovered by Respighi (1872), Pickering (1881) and Copeland (1884). The earliest wavelength lists of the emission lines were published by Campbell (1894). Wright (1918) produced further wavelength lists and provided some identifications; he also noted that the nuclei of planetary nebulae were often Wolf-Rayet stars. A major contribution was made by Plaskett (1924) who investigated all northern Wolf-Rayet stars brighter than 9th mag. He presented spectra, wavelength lists, further identifications and suggested a classification scheme. Perrine $(1920)$ and Payne $(1926,1927)$ (she was referred to as Miss Payne) extended the investigations to the southern sky and also provided additional wavelengths of emission lines.

However, the classic paper was published by Beals in 1929 entitled "On the Nature of Wolf-Rayet Emission" based on a study of Wolf-Rayet stars down to llth mag. He described the spectra as "atomic emission in the form of broad bands superposed on a comparatively faint continuous background" with half-widths $\sim 10$ to $100 \mathrm{~A}$ and a very large ratio of line to continuum intensity. He concluded that the broadening was due to the Doppler effect and 
reflected the large velocity of emitting gases in the line of sight. He ruled out other broadening agents such as Stark effect, Zeeman effect or high pressure being responsible for the large line widths. Instead he put forth the expansion hypothesis which is still with us today. He compared the Wolf-Rayet spectra with those of novae and noted that the great concentration of energy in the emission lines suggested that the origin of the emission must be the same. Also the violet-displaced absorption features on many emission lines were due to the outward motion of absorbing gases, especially He I. He proposed an expanding envelope or nebula fed by the continuous ejection of material from the central star. A constant velocity of expansion for the envelope produced a flat-topped profile and the model qualitatively accounted for all of the observed features. As for the agency driving the ejection he suggested selected radiation pressure following Milne's (1926) work in connection with the solar chromosphere where ejection velocities of the order of a few $100 \mathrm{~km} / \mathrm{sec}$ were required. Johnson (1926) had shown the importance of radiation pressure and the fact that the ratio of radiation pressure to gravity could have widely different values for different ions, e.g., He II, C III, Si IV, O III and N III had very high ratios. Hence, the intensity of emission lines also reflected large ratios of radiation pressure to gravity. Beals also estimated the mass loss $\Delta M / M \sim 4 \times 10^{-9}$ per year assuming Vejection $=2000 \mathrm{~km} / \mathrm{sec}$., $\rho=3 \times 10^{-17} \mathrm{gm} / \mathrm{cm}^{3}, R=10^{8} \mathrm{~km}$ and $M=30 \mathrm{M \theta}$. The key factors were a high surface temperature and a low surface gravity. It was also "logical to assume that Wolf-Rayet stars are very massive."

Beals and Plaskett $(1935,1938)$ under the auspices of Commission 29 of the IAU provided the basic classification scheme for Wolf-Rayet spectra that we use today: WC stars with lines of $\mathrm{C}$ and $\mathrm{O}$ and WN stars with lines of $\mathrm{N}$ with both types having lines of $\mathrm{He} I$ and He II. The scheme was based on the relative intensities of emission lines from two stages of ionization as well as the widths of the lines in the WC sequence. Beals (1940) also used the Zanstra mechanism to estimate temperatures for Wolf-Rayet stars ranging from 59,000 to $110,000^{\circ} \mathrm{K}$. This assumed an optically thin atmosphere with no collisions.

The first theoretical attempts to explain the Wolf-Rayet stars came in 1933 when Gerasimovic discussed the contours of the emission lines and in 1934 when Chandrasekhar published a paper entitled "On the Hypothesis of the Radial Ejection of High-Speed Atoms for the Wolf-Rayet Stars and the Novae." Chandrasekhar assumed an extensive envelope with radial ejection with $\mathrm{V}_{\text {ejection } ~} 500$ to $3000 \mathrm{~km} / \mathrm{sec}$. He also stated that with a dynamical theory of ejection specifying $\rho(r)$ and $v(r)$ one could calculate the emission per unit volume and hence the line profile. He considered two possible theories: 1) the atoms are repelled from the surface by some kind of force $(=\mathrm{fg})$ which falls off like gravity -- this would include Milne's radiation pressure mechanism; or 2) the atom receives a large initial outward velocity 
and is then decelerated by gravity. The velocity could be imparted by an explosion or perhaps via a mechanism suggested by Plaskett (1924), i.e., atoms near the boundary receive a large outward momentum on being ionized with the emission line being produced by subsequent recombination. Chandrasekhar calculated the expected emission line profiles from these two theories including occultation effects since the star is not of negligible dimensions with respect to the envelope. He concluded that the repulsive force model produced profiles in better agreement with those observed.

Finally, another fruitful area of Wolf-Rayet study was opened up with Wilson's 1940 paper on the spectroscopic binary HD 193576 (V444 Cygni). The spectral types were WN 5 and B1 with masses of 9.74 and $24.8 \mathrm{Mo}$, respectively. He calculated the absolute magnitude from the interstellar absorption lines of CA II H and $\mathrm{K}: \mathrm{MV}=-1.7$ and a distance of 740 parsecs. The Wolf-Rayet star was 4 mag. brighter than the absolute magnitude estimated from its mass and the standard mass-luminosity relationship. He also noted the variations in the line profile of He II 4686 as a function of phase. This work led to a series of papers on WolfRayet binaries and the general conclusions that $R * \sim 2-3 R o, \operatorname{Renv} \sim 10 R *$ and $T_{\text {surface }} \sim 50,000$ to $100,000^{\circ} \mathrm{K}$.

The rest is history! But these early astronomers and physicists were the real pioneers who reached conclusions which were not far off the mark. They made brilliant deductions with a minimum of analytic equipment or even the necessary physics. We might very well ask what could they have done with the benefit of modern day computers, detectors and technology? Who's to say? This symposium in Bali is really a tribute to these pioneers who set the field on its modern course. Van der Hucht, in his talk today, said that we have had 120 years of papers on Wolf-Rayet Stars and that was a "large number of papers under the bridge." That is true, but the first few were probably the most important and we should not forget that.

\section{References}

Beals, C. S. (1929) M.N. 90, 202.

Beals, C. S. (1930) Pub. D. A. O. 4, 271.

Beals, C. S. (1940) JRASC 34, 169.

Beals, C. S. and Plaskett , J. S. (1935) Trans. I.A.U. 5, 184.

Campbell, W. W. (1894) Astr. Ap 13, 448. 
Chandrasekhar, S. (1934) M.N. 24, 522.

Copeland, R. (1884) Copernicus 3, 206.

Gerasimovic, B. P. (1933) Z.f. Ap. Z, 335.

Johnson, M. C. (1926) M.N. 86, 300.

Kuhi, L. V. (1969) in K. B. Gebbie and R. N. Thomas (eds.), Wolf-Rayet Stars, NBS Pub. \#307, p. 101.

Kuhi, L. V. (1971) in M. K. V. Bappu and J. Sahade (eds.), Wolf-Rayet and High

Temperature Stars, I.A.U. Symposium \#49 Reidel Publ., Dordrecht, Holland, p. 205.

Milne, E. A. (1926), M.N. $86,459$.

Paczynski, B. (1971) in M. K. V. Bappu and J. Sahade (eds.), Wolf-Rayet and High

Temperature Stars, I.A.U. Symposium \#49, Reidl Publ., Dordrecht, Holland, p. 143.

Payne, C. H. (1926) H.B. \#834, 836.

Payne, C. H. (1927) H.B. \#842, 843.

Payne, C. H. (1933) Z.Ap. Z, 1, 143.

Perrine, C. D. (1920) Ap. J. 52, 39; MN 81, 142.

Pickering, W. H. (1881) Nature 23, 604.

Plaskett, J. S. (1924) Pub. D. A. O. 2, \#16.

Respighi, M. (1872) Comptes Rendues 74, 516.

Smith, L. F. (1969) in K. B. Gebbie and R. N. Thomas (eds.), Wolf-Rayet Stars, NBS Pub. $\# 307$, p. 21.

Smith, L. F. (1971) in M. K. V. Bappu and J. Sahade (eds.), Wolf-Rayet and High Temperature Stars, I.A.U. Symposium \#49, Reidl Publ., Dordrecht, Holland, p. 15.

Thomas, R. N. (1969) in K. B. Gebbie and R. N. Thomas (eds.), Wolf-Rayet Stars, NBS Pub. \#307, p. 237

Thomas, R. N. (1971) in K. B. Gebbie and R. N. Thomas (eds.), Wolf-Rayet and High Temperature Stars, NBS Pub. \#307, p. 3.

Underhill, A. B. (1969) in K. B. Gebbie and R. N. Thomas (eds.), Wolf-Rayet Stars, NBS Pub. \#307, p. 181.

Van Blerkom, D. (1971) in K. B. Gebbie and R. N. Thomas (eds.), Wolf-Rayet and High Temperature Stars, NBS Pub. \#307, p. 165.

Wilson, O. C. (1940) Ap. J. $21,379$.

Wolf, C. J. E., and Rayet, G. (1867) Comptes Rendues 65, 292.

Wright, W. H. (1918) Lick Pub. 13, 224. 


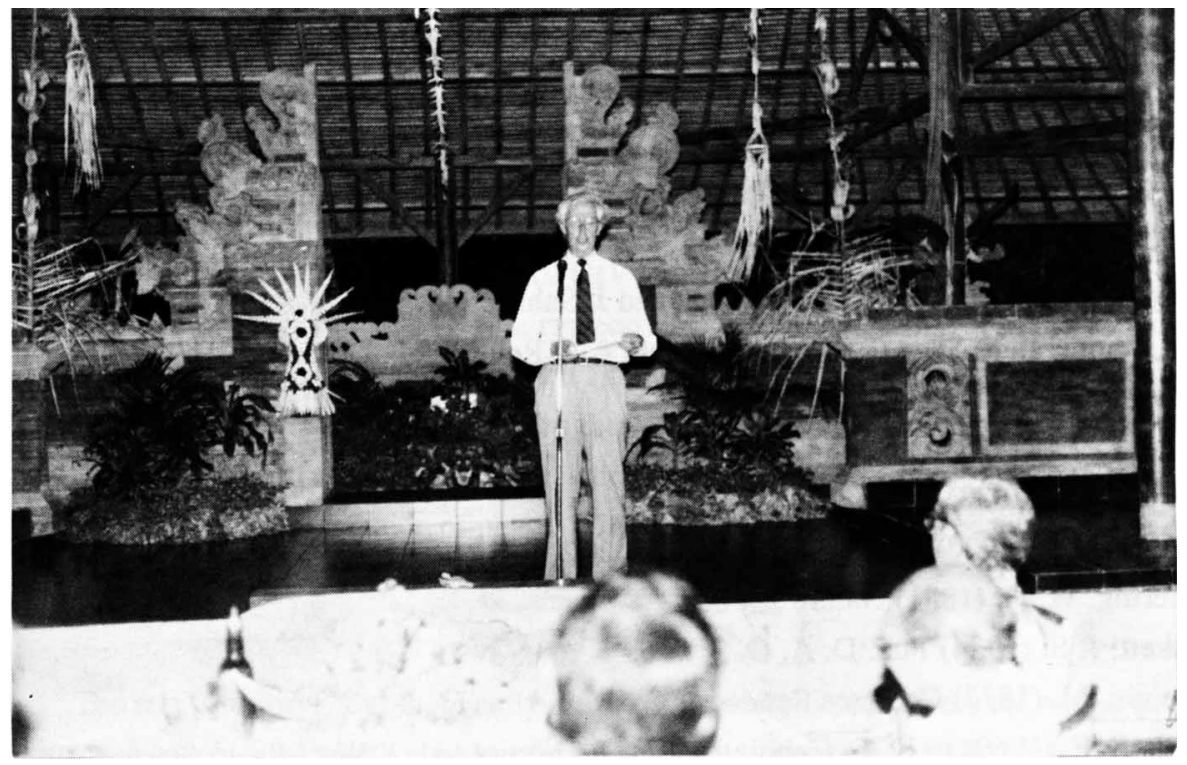

Len Kuhi 